Supporting Information

\title{
Wood-Nanotechnology-Based Membrane for the Efficient Purification of Oil-in-Water Emulsions
}

Seongmin Kim, Kihwan Kim, Gyosik Jun, and Woonbong Hwang *

Department of Mechanical Engineering, Pohang University of Science and Technology, Pohang, Gyeongbuk, 37673, Republic of Korea

* Corresponding author

Email:whwang@postech.ac.kr 


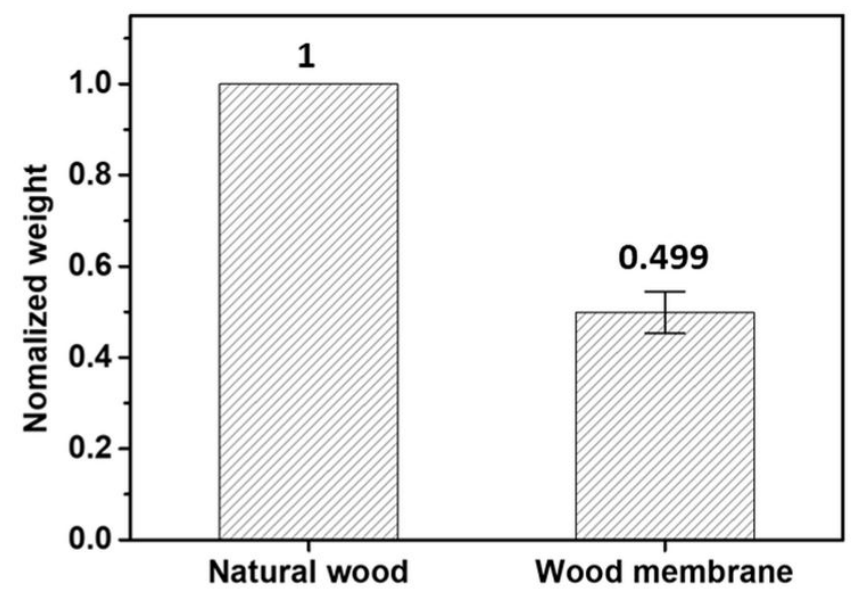

Figure S1. Weights of wood samples before and after top-down chemical treatment. 


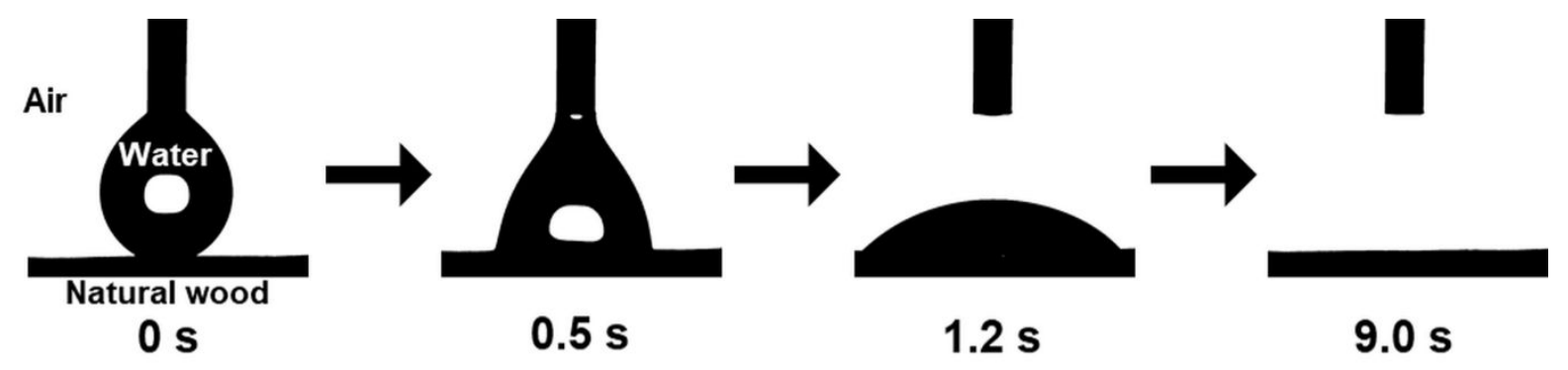

Figure S2. Wetting characteristics of natural wood. 


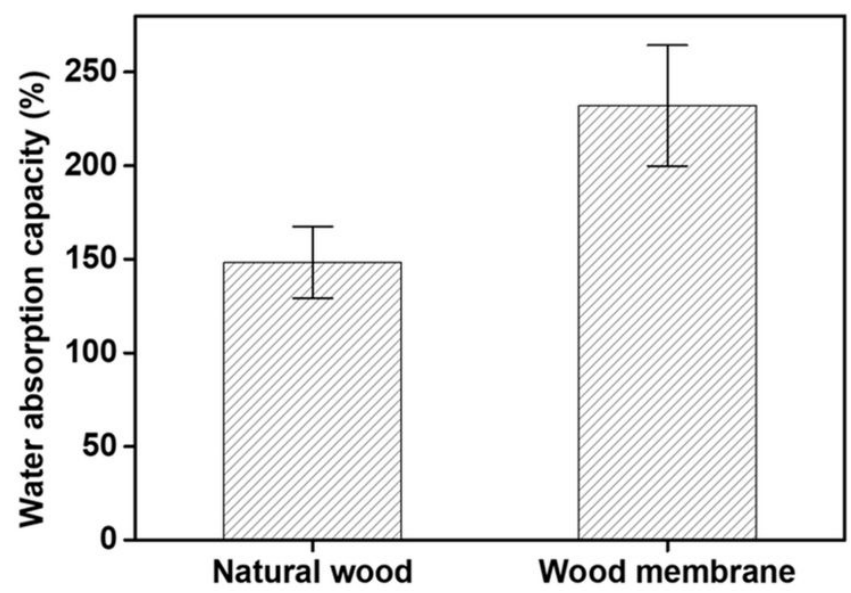

Figure S3. Water absorption capacities of wood samples before and after top-down chemical treatment. 

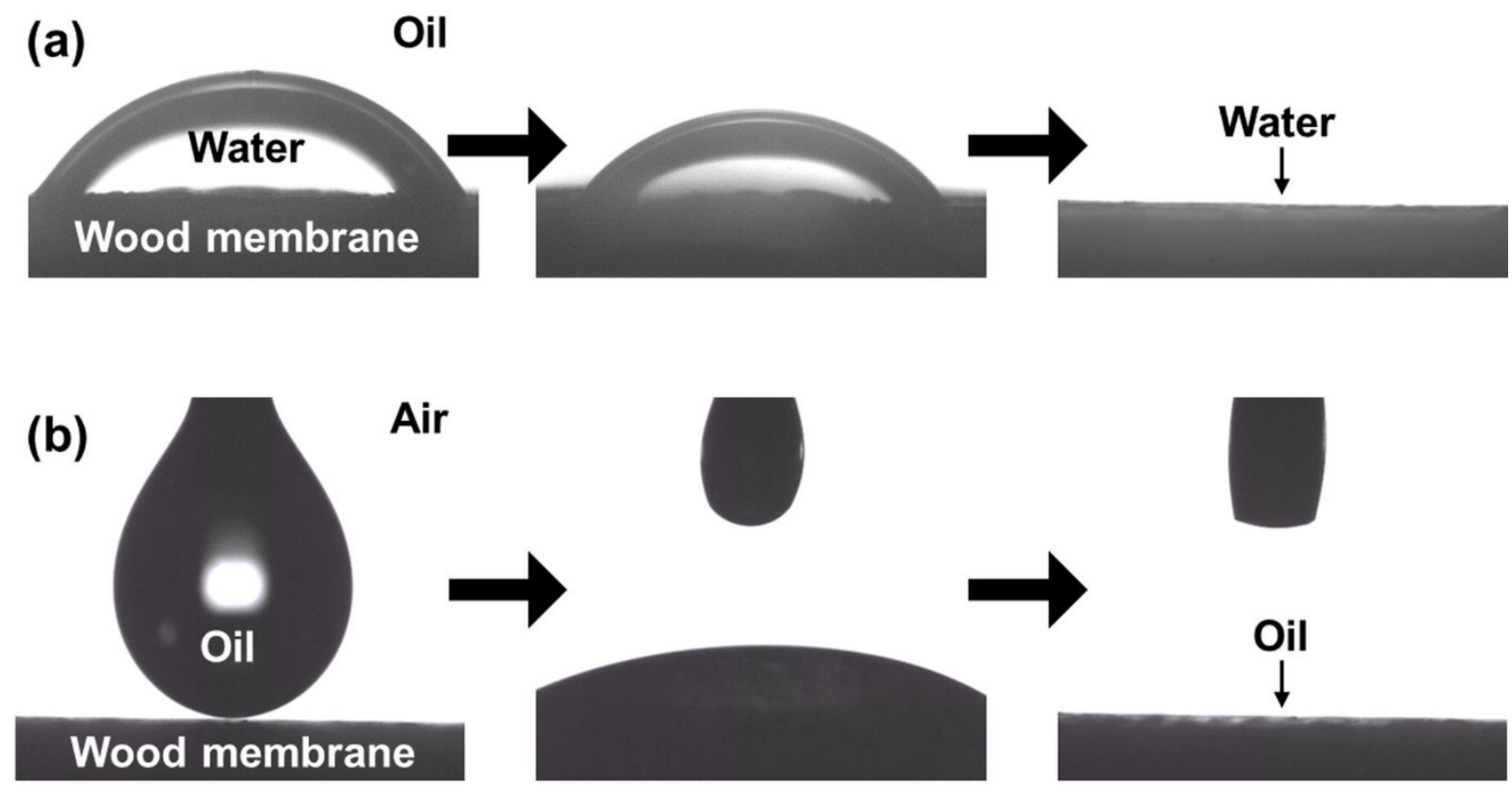

Figure S4. Photographic images showing (a) the water-absorbing properties of the wood membrane under oil, and (b) the oil-absorbing properties of the wood membrane in air. 

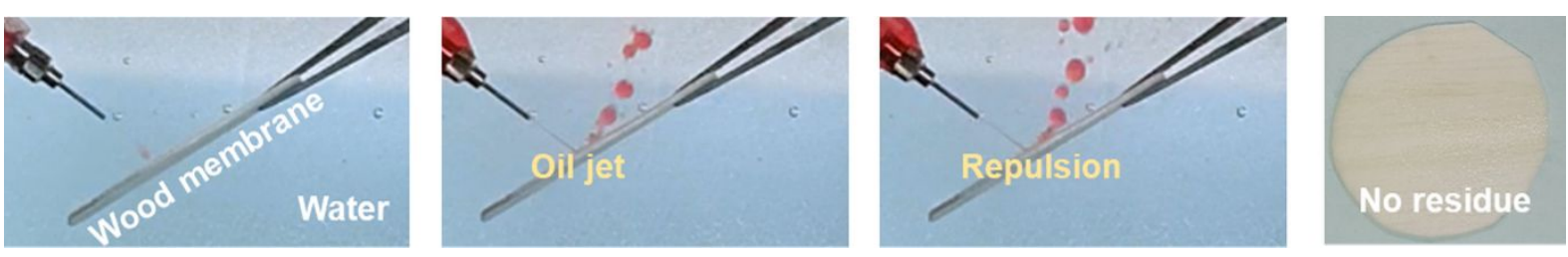

Figure S5. Photographic images showing the underwater anti-oil properties of the wood membrane. 


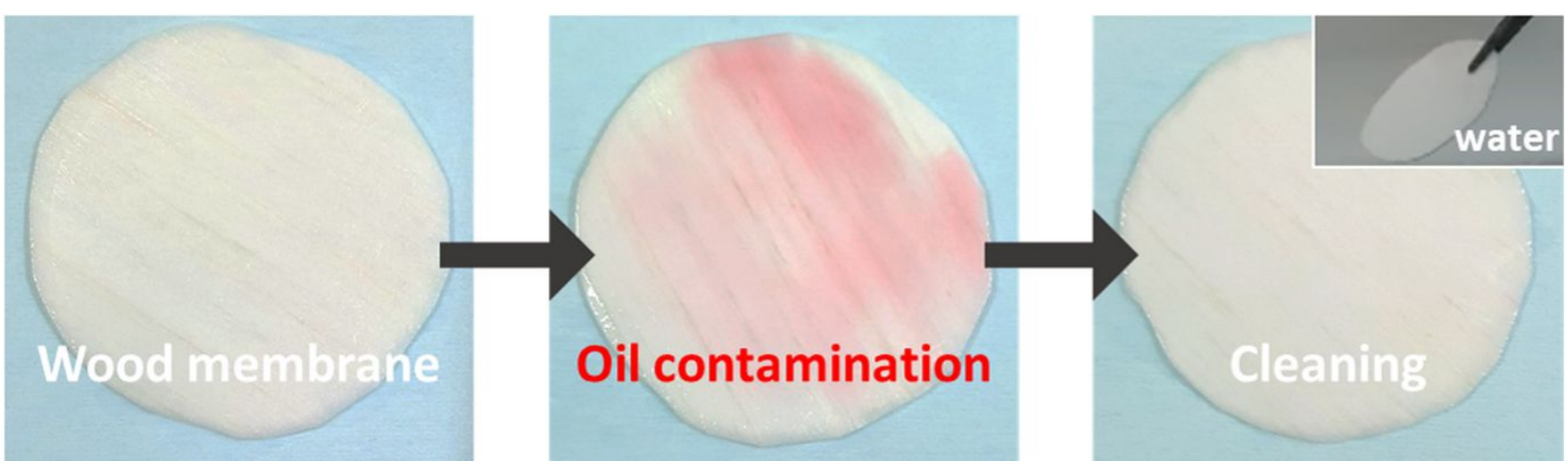

Figure S6. Photographic images showing the self-cleaning properties of the wood membrane. 


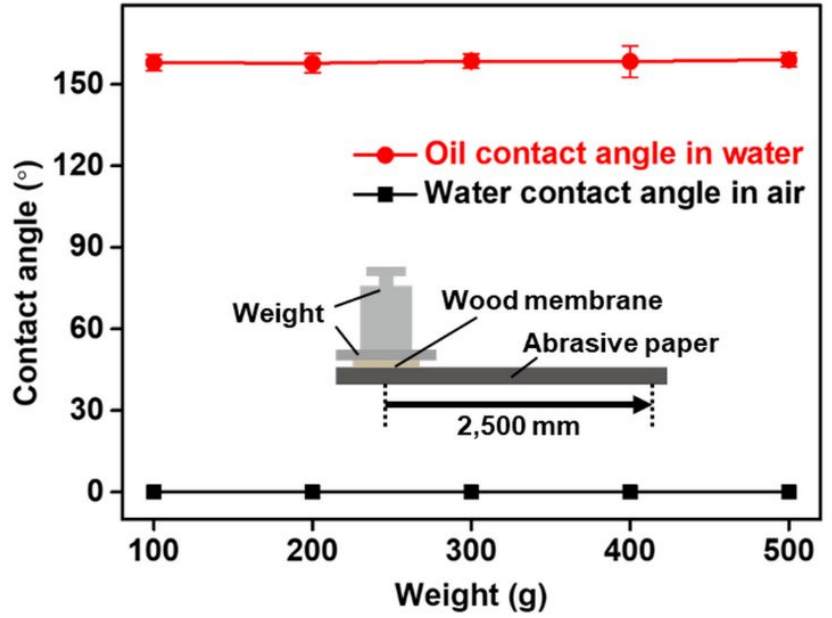

Figure S7. Wetting characteristics of the wood membrane after 2,500 $\mathrm{mm}$ of abrasion under various loads. 

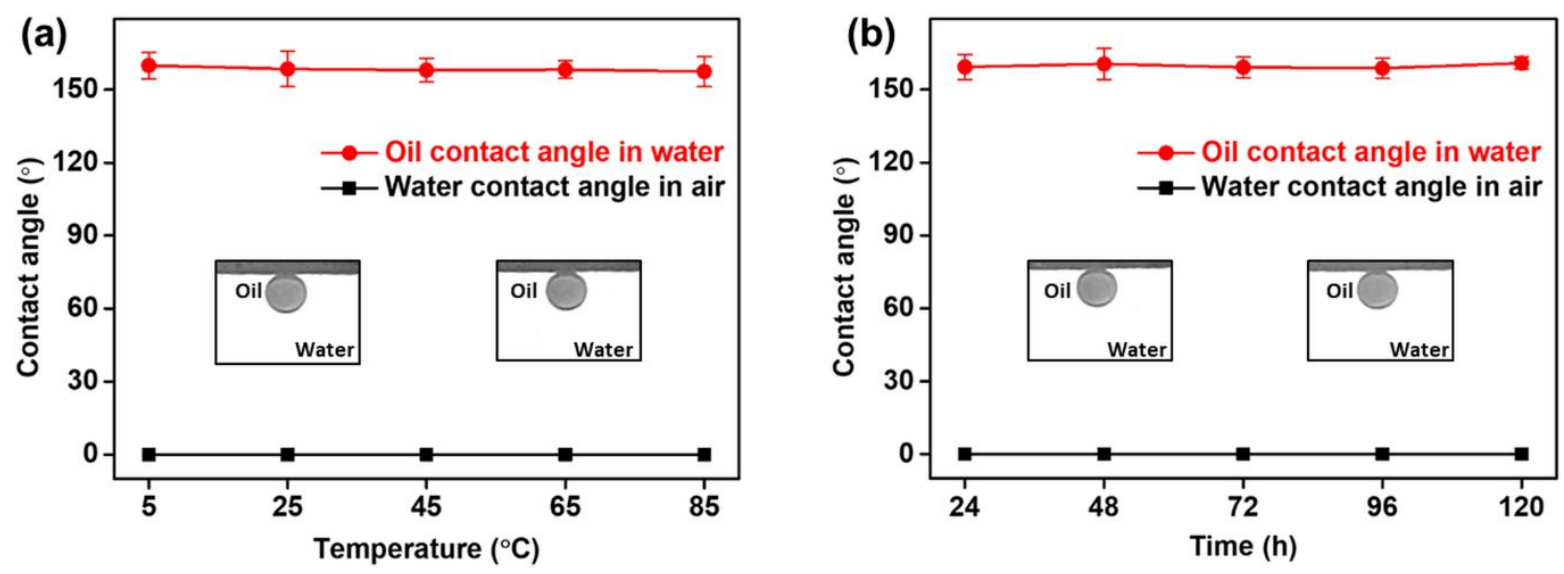

Figure S8. Durability of the wood membrane. Wetting characteristics (a) after immersion in cold-to-hot water for $168 \mathrm{~h}$ and (b) during UV irradiation for $120 \mathrm{~h}$. 

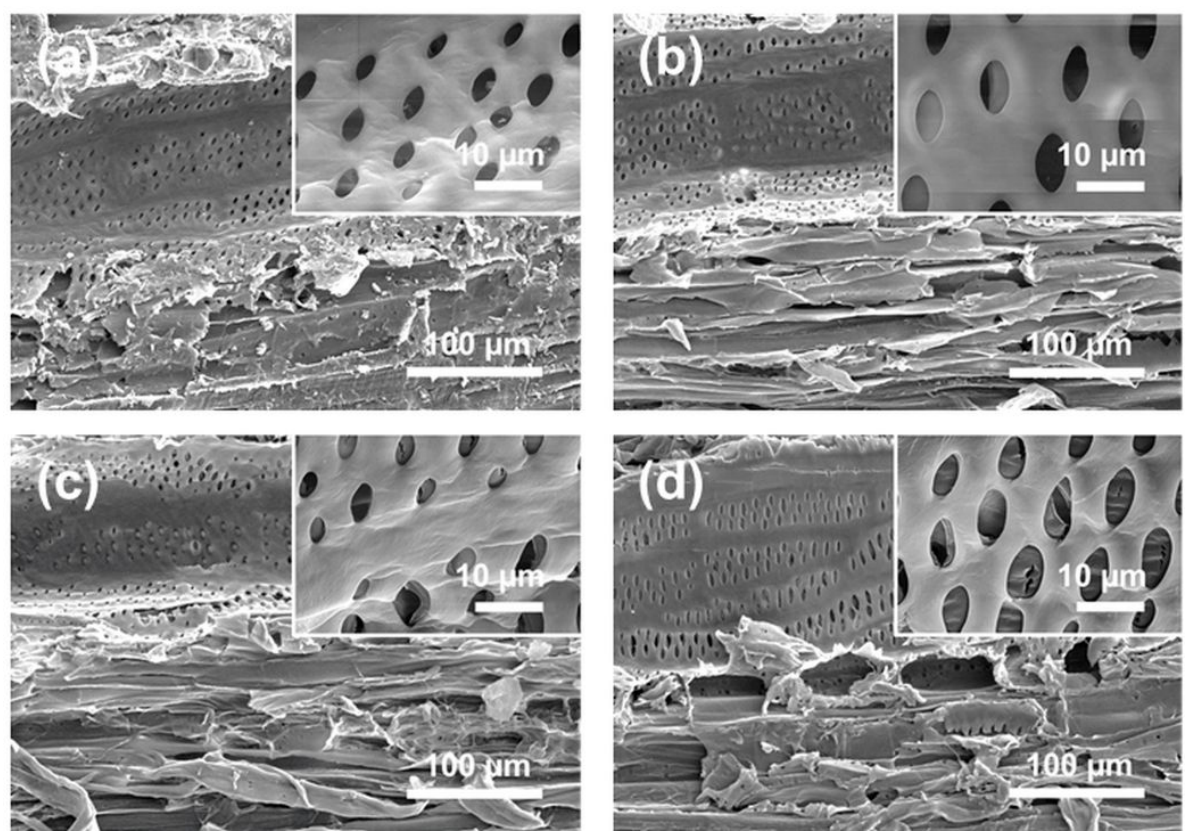

Figure S9. SEM images of the wood membrane after (a) 2,500 $\mathrm{mm}$ of abrasion under $225 \mathrm{~g}$ load,

(b) $168 \mathrm{~h}$ of immersion in a pH 3 solution, (c) $168 \mathrm{~h}$ of immersion in a $\mathrm{pH} 11$ solution, and (d) $120 \mathrm{~h}$ of UV irradiation. 


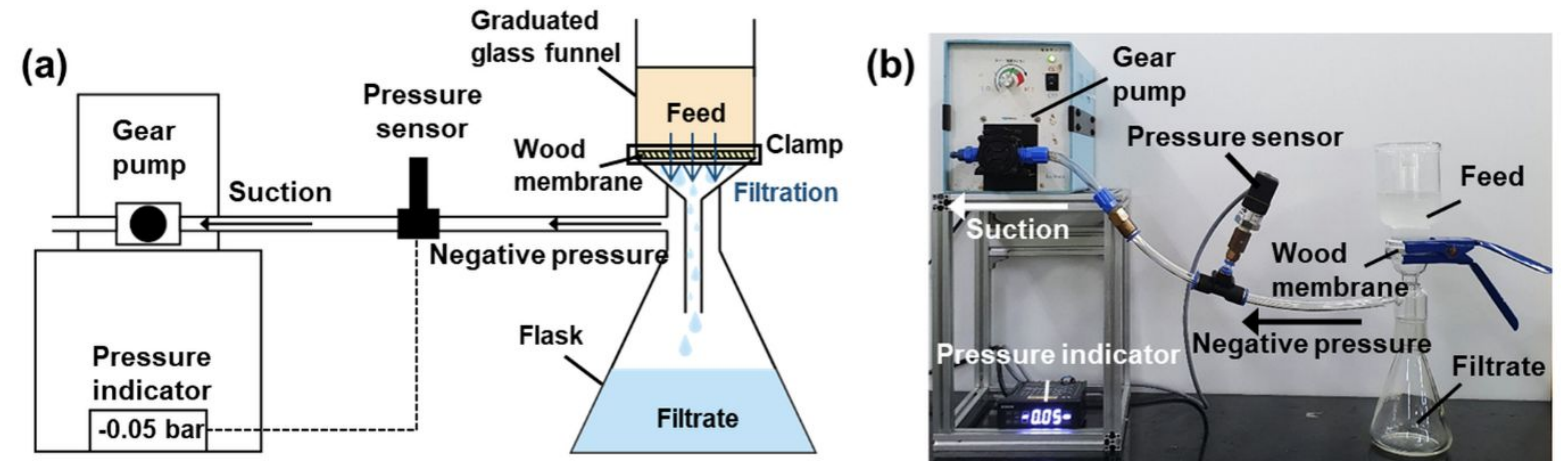

Figure S10. Experimental setup for emulsion-separation testing: (a) Schematic illustration, and (b) photographic image. 


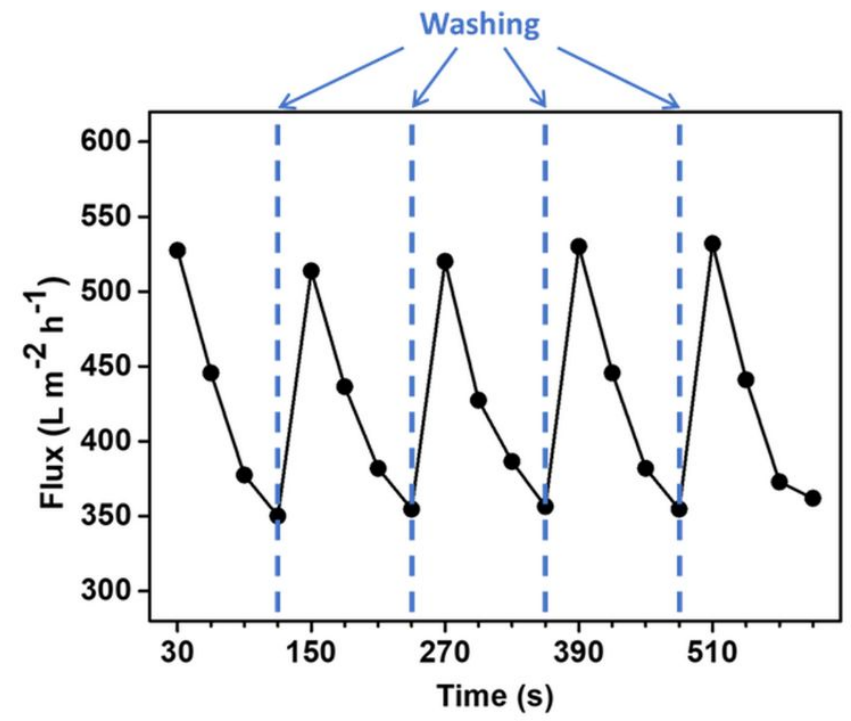

Figure S11. Changes in filtration flux over time during emulsion-separation testing. 


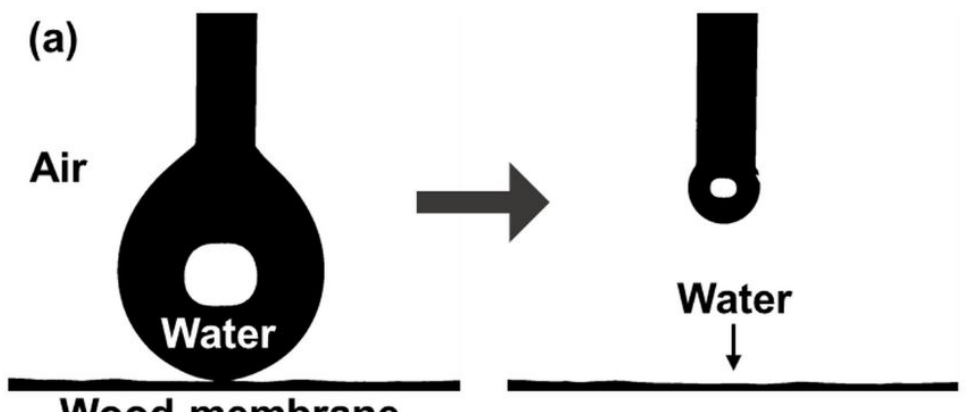

(b)

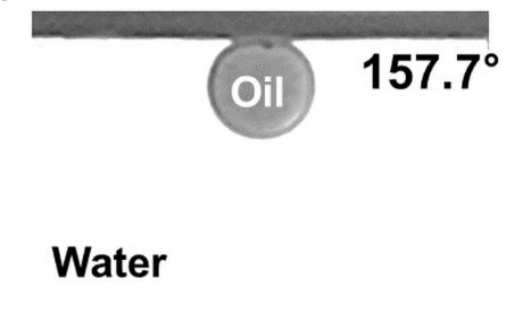

Figure S12. Wetting characteristics of the wood membrane after 10 cycles of emulsionseparation use. 
Table S1. Highlighting the cost-effectiveness of the wood material: comparing the costs of various materials.

\begin{tabular}{|c|c|c|c|c|}
\hline Type & Purchase rink & Dimension & Price & Cost $\left(\$ / \mathrm{m}^{2}\right)$ \\
\hline $\begin{array}{l}\text { Nylon } \\
\text { membrane }\end{array}$ & $\begin{array}{l}\text { https://www.amazon.com/Tisch-Brand-Membrane-Filter-5- } \\
\text { 00um/dp/B01B4YEU42/ref=sr_1_4?dchild=18keywords=tisch+membrane+filter\&qid=1591708333\&sr=8-4 }\end{array}$ & dia., $47 \mathrm{~mm}$ & $\begin{array}{l}\$ 119.13 / \\
200 \text { pcs }\end{array}$ & 343.5 \\
\hline $\begin{array}{l}\text { Polyethylene } \\
\text { membrane }\end{array}$ & $\begin{array}{l}\text { https://www.amazon.com/Tisch-Polytetrafluoroethylene-Membrane-Filter- } \\
\text { Hydrophobic/dp/B01B4YDSR2/ref=s____? ?dchild=1\&keywords=tisch+membrane+filter\&qid=1591708333\&sr=8-7 }\end{array}$ & dia., $47 \mathrm{~mm}$ & $\begin{array}{l}\$ 104.24 / \\
100 \text { pcs }\end{array}$ & 601.1 \\
\hline Copper mesh & $\begin{array}{l}\text { https://www.amazon.com/Beyondsupply-100-Copper-Micron-Filter- } \\
\text { Screen/dp/B074DPW8XT/ref=sr_1_1?dchild=18keywords=woven+wire+mesh\&qid=1597222187\&refinements=p_n_feature_seven_ } \\
\text { browse-bin\%3A5485421011\&refresh=2\&rnid=3071213011\&sr=8-1 }\end{array}$ & $12 \times 48$ inch & $\$ 55.00$ & 148.0 \\
\hline $\begin{array}{l}\text { Stainless steel } \\
\text { mesh }\end{array}$ & 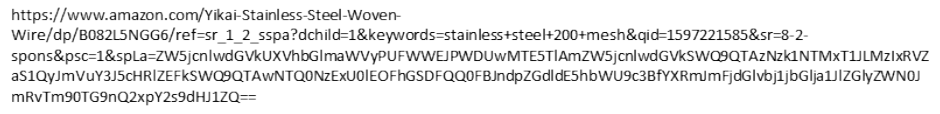 & $300 \times 1000 \mathrm{~mm}$ & $\$ 39.99$ & 133.3 \\
\hline $\begin{array}{l}\text { Balsa wood } \\
\text { sheet }\end{array}$ & https://www.amazon.com/Viloga-Sheets-Thickness-Wooden-Aircraft/dp/B07HHCGCNW?th=1 & $100 \times 500 \mathrm{~mm}$ & $\begin{array}{l}\$ 18.99 / \\
10 p c s\end{array}$ & 37.98 \\
\hline
\end{tabular}

